\title{
On Fuzzy Fractional Laplace Transformation
}

\author{
Ahmad Jafarian, ${ }^{1}$ Alireza Khalili Golmankhaneh, ${ }^{2}$ and Dumitru Baleanu ${ }^{3,4,5}$ \\ ${ }^{1}$ Department of Mathematics, Urmia Branch, Islamic Azad University, P.O. Box 969, Oromiyeh, Iran \\ ${ }^{2}$ Department of Physics, Urmia Branch, Islamic Azad University, P.O. Box 969, Oromiyeh, Iran \\ ${ }^{3}$ Department of Chemical and Materials Engineering, Faculty of Engineering, King Abdulaziz University, Jeddah, Saudi Arabia \\ ${ }^{4}$ Department of Mathematics and Computer Science, Çankaya University, 06530 Ankara, Turkey \\ ${ }^{5}$ Institute of Space Sciences, P.O. Box, MG-23, 76900 Magurele-Bucharest, Romania
}

Correspondence should be addressed to Alireza Khalili Golmankhaneh; alirezakhalili2002@yahoo.co.in

Received 12 February 2014; Accepted 3 March 2014; Published 30 March 2014

Academic Editor: Xiao-Jun Yang

Copyright (C) 2014 Ahmad Jafarian et al. This is an open access article distributed under the Creative Commons Attribution License, which permits unrestricted use, distribution, and reproduction in any medium, provided the original work is properly cited.

Fuzzy and fractional differential equations are used to model problems with uncertainty and memory. Using the fractional fuzzy Laplace transformation we have solved the fuzzy fractional eigenvalue differential equation. By illustrative examples we have shown the results.

\section{Introduction}

Fractional calculus is the generalization of the standard calculus. That involves the derivative of functions to arbitrary orders. But the fractional derivatives are nonlocal so they provided the mathematical models to non-Markov processes and memory processes. Fractional calculus has found many applications in science, engineering, and so forth [1-15]. Fractional dynamics has been introduced and it can be one of the models for the nonconservative systems. Fractional Newtonian with the memory is modeled by heterogeneous liquid [10]. Recently, fractional local derivative has been studied and generalized so that it can be applied on fractals [16]. Local fractional calculus and application in science and engineering have been suggested on Cantor sets [17, 18]. The uncertainty is important subject in measurement of quantities in physics. Fuzzy number can be used to show the uncertainty in measurement. Fuzzy sets have been introduced by Lotfi Zadeh in 1965 and since then they have been used in many applications [19-26]. As a consequence, there is vast literature on the practical applications of fuzzy sets, while theory has a more modest coverage. Fuzzy fractional heat and wave equation has been solved by using homotopy analysis transform method [27]. This paper adopted fuzzy Laplace transforms method to solve problems of fuzzy fractional differential equations. Our motivation in this paper is due to two reasons. Firstly, one of the important and interesting transforms in the problems of fuzzy equations is Laplace transforms. The fuzzy Laplace transform method solves fuzzy fractional differential equations and fuzzy boundary and initial value problems [28-35]. Secondly, this method is practically the most important operational method and also has advantage that it solves problems directly without determining a general solution in the first step and developing nonhomogeneous differential equation in the second step.

This paper is arranged in the following manner.

After an introduction to the present work, in Section 2, we recall some basic tools that involve the fractional calculus and the fuzzy numbers. In Section 3, the fuzzy fractional Laplace transformation is discussed. Finally, we present the conclusions in Section 4.

\section{Basic Tools}

2.1. Fractional Calculus. Fractional calculus deals with generalizations of integer order derivatives integrals to arbitrary order. In this section we present basic definitions and properties which will be used in the subsequent sections [1-13]. If $f(x) \in C[a, b]$ and $\alpha>0$, then

$$
\begin{gathered}
{ }_{a} I_{x}^{\alpha} f(x):=\frac{1}{\Gamma(\alpha)} \int_{a}^{x} \frac{f(t)}{(x-t)^{1-\alpha}} d t \\
{ }_{a} D_{x}^{\alpha} f(x):=\frac{1}{\Gamma(n-\alpha)}\left(\frac{d}{d x}\right)^{n} \int_{a}^{x} \frac{f(t)}{(x-t)^{-n+\alpha+1}} d t
\end{gathered}
$$




$$
\begin{gathered}
{ }_{x}^{C} D_{b}^{\alpha} f(x)=\frac{1}{\Gamma(n-\alpha)} \int_{x}^{b}(t-x)^{n-\alpha-1}\left(-\frac{d}{d t}\right)^{n} f(t) d t, \\
{ }_{a} I_{t \quad a}^{\alpha} D_{t}^{\alpha} x(t)=x(t)-\sum_{j=1}^{n} \frac{\left({ }_{a} D_{t}^{\alpha-j} x\right)(a)}{\Gamma(\alpha+1-j)}(t-a)^{\alpha-j}
\end{gathered}
$$

are called the left sided Riemann-Liouville (RL), fractional integral Riemann-Liouville, fractional derivative of order $\alpha$, and left sided Caputo fractional derivatives, respectively.

\subsection{Fuzzy Numbers}

Definition 1. A fuzzy number is a fuzzy set $u: \mathbb{R}^{1} \rightarrow I=$ $[0,1]$ such that

(i) $u$ is upper semicontinuous;

(ii) $u(x)=0$ outside some interval $[a, d]$;

(iii) there are real numbers $b$ and $c, a \leq b \leq c \leq d$, for which

(1) $u(x)$ is monotonically increasing on $[a, b]$,

(2) $u(x)$ is monotonically decreasing on $[c, d]$,

(3) $u(x)=1, b \leq x \leq c$.

The set of all the fuzzy numbers (as given in Definition 1) is denoted by $E^{1}$ [19-26].

Definition 2. A fuzzy number $v$ is a pair $(\underline{v}, \bar{v})$ of functions $\underline{v}(r)$ and $\bar{v}(r), 0 \leq r \leq 1$, which satisfy the following requirements:

(i) $\underline{v}(r)$ is a bounded monotonically increasing, left continuous function on $(0,1]$ and right continuous at 0

(ii) $\bar{v}(r)$ is a bounded monotonically decreasing, left continuous function on $(0,1]$ and right continuous at 0 ;

(iii) $\underline{v}(r) \leq \bar{v}(r), 0 \leq r \leq 1$.

A popular fuzzy number is the triangular fuzzy number $v=\left(v_{m}, v_{l}, v_{u}\right)$, where $v_{m}$ denotes the modal value and the real values $v_{l} \geq 0$ and $v_{u} \geq 0$ represent the left and right fuzziness, respectively. The membership function of a triangular fuzzy number is defined as follows:

$$
\mu_{v}(x)= \begin{cases}\frac{x-v_{m}}{v_{l}}+1, & v_{m}-v_{l} \leq x \leq v_{m}, \\ \frac{v_{m}-x}{v_{u}}+1, & v_{m} \leq x \leq v_{m}+v_{u}, \\ 0, & \text { otherwise. }\end{cases}
$$

Its parametric form is

$$
\begin{gathered}
\underline{v}(r)=v_{m}+v_{l}(r-1), \\
\bar{v}(r)=v_{m}+v_{u}(1-r), \quad 0 \leq r \leq 1 .
\end{gathered}
$$

Triangular fuzzy numbers are fuzzy numbers in $L R$ representation, where the reference functions $L$ and $R$ are linear.

\section{Fuzzy Fractional Laplace Transformation}

Initial value problems are considered in fractional differential equations and solved by analytical and numerical methods [12]. In recent works, dynamical processes are considered the randomness and uncertainty. Stochastic and fuzzy differential equations are mathematical model for such dynamical processes, respectively. Suppose $\alpha \in(0,1], G>0$ and $F$ is fuzzy real number [24]. Then, the fractional fuzzy differential is

$$
{ }_{0} D_{t}^{\alpha} x(t)=f(t, x(t)), \quad t \in(0, G], x_{0} \in F,
$$

where $f[0, G] \times F \rightarrow F$ is continuous in the case of $f[0, G] \times$ $R \rightarrow R$ and $x_{0} \in R$ so (4) reduces to a fractional differential equation. And if one chooses $\alpha=1$ in (4), we have a fuzzy differential equation.

3.1. Fractional Differential Equations with Uncertainty. Let us consider the fractional equation with fuzzy condition; that is,

$$
\begin{gathered}
{ }_{0} D_{t}^{\alpha} x(t)=f(t, x(t)), \\
\lim _{t \rightarrow 0^{+}} t^{1-\alpha} x(t)=x_{0} \in F \quad t \in(0, G] \quad x_{0} \in F,
\end{gathered}
$$

where $f:[0, G] \times F \rightarrow F$ is continuous [24]. For example, if $\lambda>0$ and $f(t, x)=\lambda x+g(t, x)$ with $g:[0, G] \times F \rightarrow F$ which is continuous, then the solution for (5) is

$$
\begin{aligned}
x(t)= & \Gamma(\alpha) t^{\alpha-1} E_{\alpha, \alpha}\left(\lambda t^{\alpha}\right) x_{0} \\
& +\int_{0} t(t-s)^{\alpha-1} E_{\alpha, \alpha}\left(\lambda(t-s)^{\alpha}\right) g(s, x(s)) d s,
\end{aligned}
$$

where

$$
E_{\alpha, \alpha}(z)=\sum_{k=0}^{\infty} \frac{z^{\alpha}}{\Gamma(\alpha(k+1))} .
$$

As a pursuit of fractional fuzzy differential in the following section we generalized fuzzy Laplace transformation method to fractional fuzzy Laplace method. Now, we solve illustrated examples in the subsequence sections.

Example 3. Consider the following fuzzy fractional eigenvalue differential equations as

$$
\begin{gathered}
D_{0^{+}}^{\alpha} y(x)+\lambda y(x)=0 \\
x \in R^{+} \cup 0, \lambda \in R^{+}, \alpha \in(0,1) \\
I_{0^{+}}^{1-\alpha} y\left(0^{+}\right)=\alpha_{\mathrm{RL}} \quad \alpha_{\mathrm{RL}}=(1+r, 3-r),
\end{gathered}
$$

where $\alpha_{\mathrm{RL}}$ is the number of fuzzy triangular which is called fuzzy Riemann-Liouville initial condition. Then, the above equation is extended based on its lower and upper functions as follows:

$$
\begin{gathered}
\left(D_{0^{+}}^{\alpha} \underline{y}(x), D_{0^{+}}^{\alpha} \bar{y}(x)\right)+\lambda(\underline{y}(x), \bar{y}(x))=0 \\
I_{0^{+}}^{1-\alpha}\left(\underline{y}\left(0^{+}\right), \bar{y}\left(0^{+}\right)\right)=(1+r, 3-r) .
\end{gathered}
$$


Now, we solve these equations according to the two following cases, using the generalized fractional fuzzy Laplace transform (FFLT). The equation with lower functions is

$$
\begin{gathered}
D_{0^{+}}^{\alpha} \underline{y}(x, r)+\lambda(\underline{y}(x, r))=0 \\
I_{0^{+}}^{1-\alpha}\left(\underline{y}\left(0^{+}, r\right)\right)=1+r
\end{gathered}
$$

and with upper functions is

$$
\begin{gathered}
D_{0^{+}}^{\alpha} \bar{y}(x, r)+\lambda \bar{y}(x, r)=0 \\
I_{0^{+}}^{1-\alpha} \bar{y}\left(0^{+}, r\right)=3-r .
\end{gathered}
$$

Now, we use the FFLT for solving (9):

$$
\begin{gathered}
D_{0^{+}}^{\alpha} \underline{y}(x, r)+\lambda \underline{y}(x, r)=0 \\
I_{0^{+}}^{1-\alpha}\left(\underline{y}\left(0^{+}, r\right)\right)=1+r .
\end{gathered}
$$

After using Laplace transform on (12), we get

$$
\begin{gathered}
S^{\alpha} \underline{Y}(s, r)-I_{0^{+}}^{1-\alpha} y\left(0^{+}, r\right)+\lambda \underline{Y}(s, r)=0, \\
\left(S^{\alpha}+\lambda\right) \underline{Y}(s, r)=(1+r) .
\end{gathered}
$$

Thus, we have

$$
\underline{Y}(s, r)=\frac{(1+r)}{S^{\alpha}+\lambda} .
$$

Taking the fuzzy inverse Laplace transform we obtain

$$
\underline{Y}(x, r)=(1+r) \cdot \exp \left(-\lambda x^{\alpha}\right) .
$$

In a similar manner we are led to

$$
\bar{Y}(x, r)=(3-r) \cdot \exp \left(-\lambda x^{\alpha}\right) .
$$

Therefore, the general solution will be

$$
\psi=(\underline{Y}(x, r), \bar{Y}(x, r)) .
$$

In Figures 1, 2, 3, and 4 we have plotted the solutions for the case of fractional, fractional fuzzy, and fuzzy, respectively.

Example 4. Let us consider the following fuzzy fractional differential equation as

$$
\begin{gathered}
D_{0^{+}}^{\alpha} y(x)+\lambda y(x)=0 \quad x \in R^{+} \cup 0, \lambda \in R^{+}, \alpha \in(0,1) \\
y(0)=(1-r, 3+r),
\end{gathered}
$$

where ${ }_{0} D_{x}^{\alpha}=D_{0^{+}}^{\alpha}$ and (18) is called fuzzy Caputo initial condition. Then, (18) will be equivalent to

$$
\begin{gathered}
D_{0^{+}}^{\alpha} \underline{y}(x, r)+\lambda \underline{y}(x, r)=0 \\
\underline{y}(0, r)=1+r, \\
D_{0^{+}}^{\alpha}(\bar{y}(x, r))+\lambda \bar{y}(x, r)=0 \\
\bar{y}(0, r)=3-r .
\end{gathered}
$$

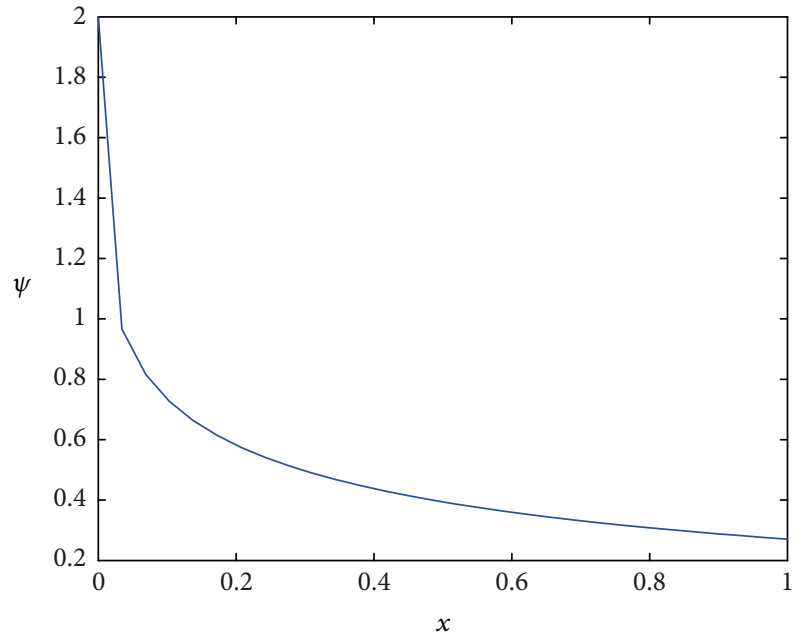

Figure $1: \alpha=0.3$ and $\lambda=2$.

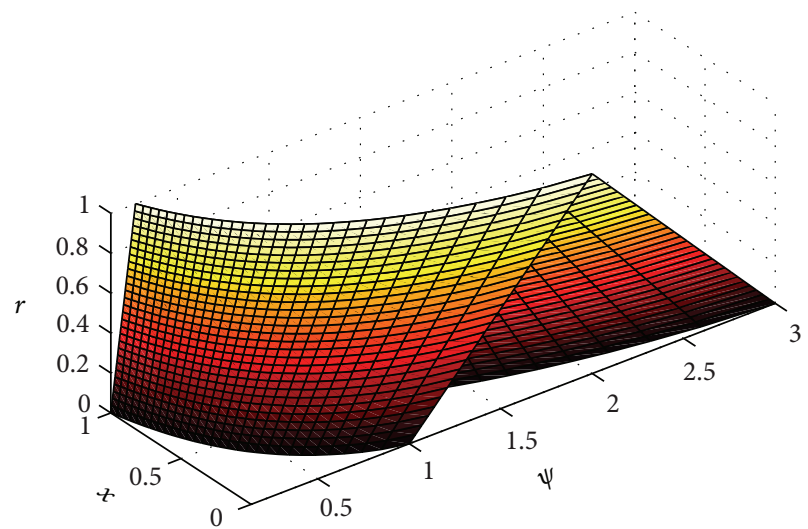

Figure 2: $\alpha=0.8$ and $\lambda=2$.

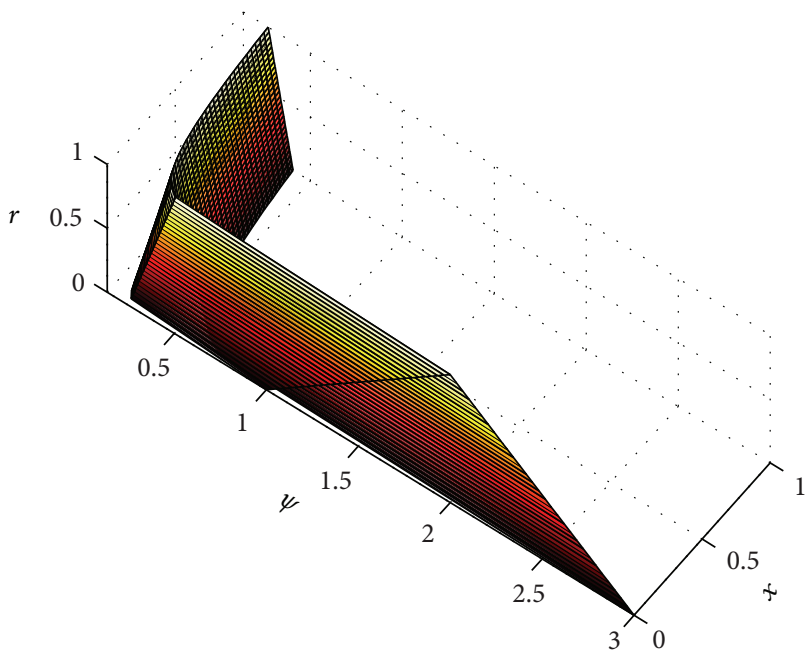

FIgURE 3: $\alpha=0.1$ and $\lambda=2$. 


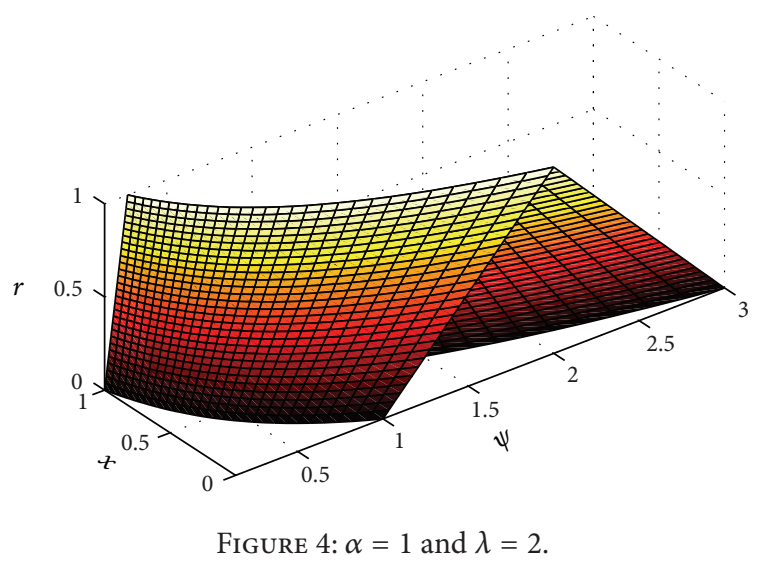

Applying Laplace transform on (19), we obtain

$$
\begin{gathered}
S^{\alpha} \underline{Y}(s, r)-S^{\alpha-1} \underline{Y}(0, r)+\lambda \underline{Y}(s, r)=0, \\
\left(S^{\alpha}+\lambda\right) \underline{Y}(s, r)-S^{\alpha-1}(1+r)+\lambda \underline{y}(s, r)=(1+r) .
\end{gathered}
$$

In view of (21) we arrive at

$$
\underline{Y}(s, r)=\frac{S^{\alpha-1}(1+r)}{S^{\alpha}+\lambda} .
$$

Also, by taking inverse Laplace transform of (22) we deduce that

$$
\underline{y}(x, r)=(1+r) E_{\alpha, 1}\left(-\lambda x^{\alpha}\right) .
$$

Likewise, by doing the same calculation (20) will be

$$
\bar{y}(x, r)=(3-r) E_{\alpha, 1}\left(-\lambda x^{\alpha}\right) .
$$

Therefore, we have the final solution

$$
\psi=(\underline{Y}(x, r), \bar{Y}(x, r)) .
$$

In Figures 5, 6, 7, and 8 we have sketched the solutions for the case of fractional, fractional fuzzy, and fuzzy, respectively.

Example 5. Consider the following fuzzy fractional differential equations with fuzzy Caputo initial condition as

$$
\begin{gathered}
D^{2} y(x)+\lambda D_{0^{+}}^{\alpha} y(x)=0, \\
x \in R^{+} \cup 0, \lambda \in R^{+}, \alpha \in(0,1) \\
y(0)=(1-r, 3+r) \\
y^{\prime}(0)=(4+r, 6-r) .
\end{gathered}
$$

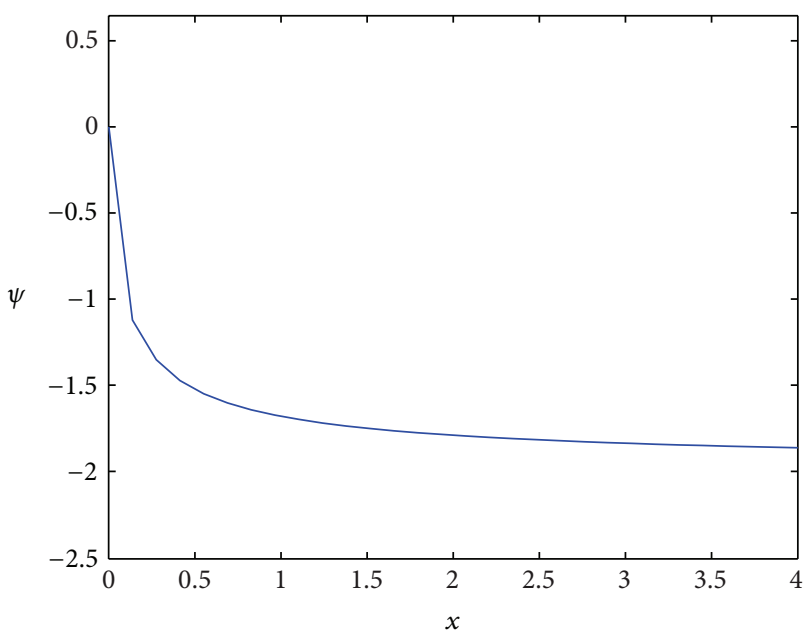

FIGURE 5: $\alpha=0.3$ and $\lambda=2$.

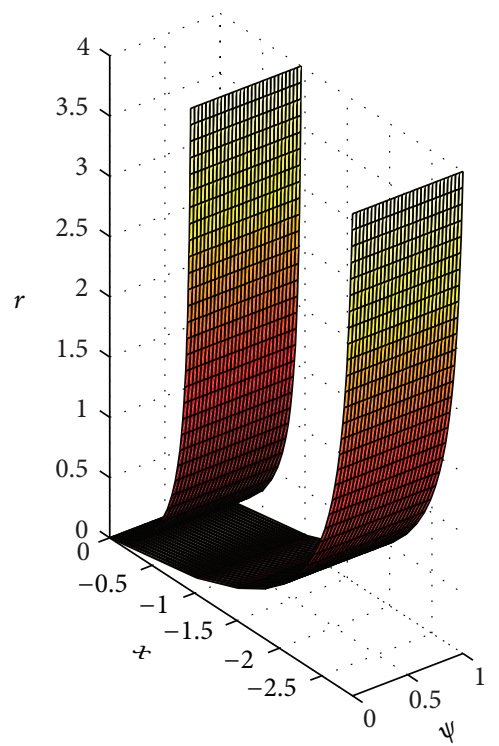

FIgURE $6: \alpha=0.8$ and $\lambda=2$.

So (26) will become two equations with lower and upper functions such as

$$
\begin{gathered}
D^{2} \underline{y}(x, r)+\lambda^{l} D_{0^{+}}^{\alpha} \underline{y}(x, r)=0 \\
\underline{y}(0, r)=1+r \\
\underline{y^{\prime}}(0, r)=4+r, \\
D^{2} \bar{y}(x, r)+\lambda^{l} D_{0^{+}}^{\alpha} \bar{y}(x, r)=0 \\
\bar{y}(0, r)=3-r \\
\overline{y^{\prime}}(0, r)=6-r .
\end{gathered}
$$




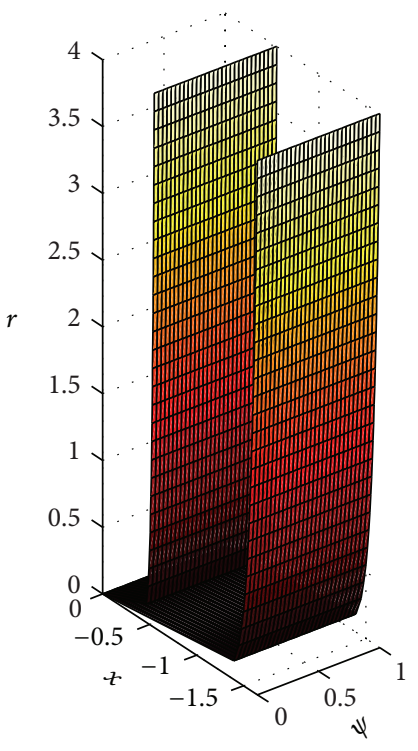

FIGURE 7: $\alpha=0.1$ and $\lambda=2$.

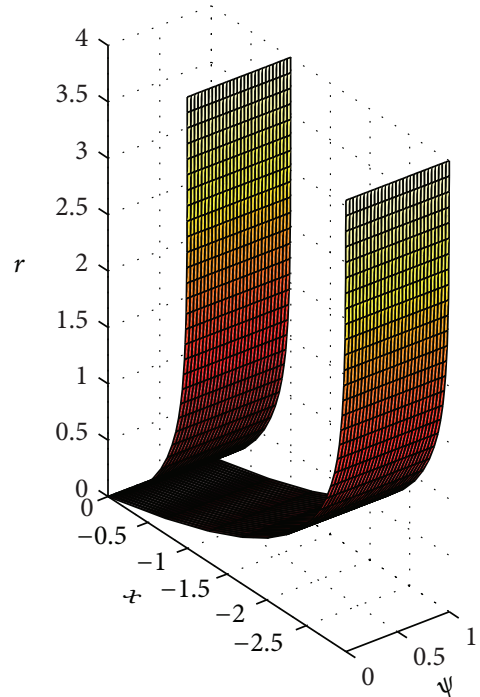

FIGURE $8: \alpha=1$ and $\lambda=2$.

Applying Laplace transform and inverse Laplace transform on (27) one is led to

$$
\begin{aligned}
\underline{y}(x, r)= & (1+r) E_{2-\alpha, 1}\left(-\lambda x^{2-\alpha}\right) \\
& +(1+r) x^{2-\alpha} E_{2-\alpha, 3-\alpha}\left(-\lambda x^{2-\alpha}\right) \\
& +(4+r) x E_{2-\alpha, 2}\left(-\lambda x^{2-\alpha}\right), \\
\bar{y}(x, r)= & (3-r) E_{2-\alpha, 1}\left(-\lambda x^{2-\alpha}\right) \\
& +(3-r) x^{2-\alpha} E_{2-\alpha, 3-\alpha}\left(-\lambda x^{2-\alpha}\right) \\
& +(6-r) x E_{2-\alpha, 2}\left(-\lambda x^{2-\alpha}\right) .
\end{aligned}
$$

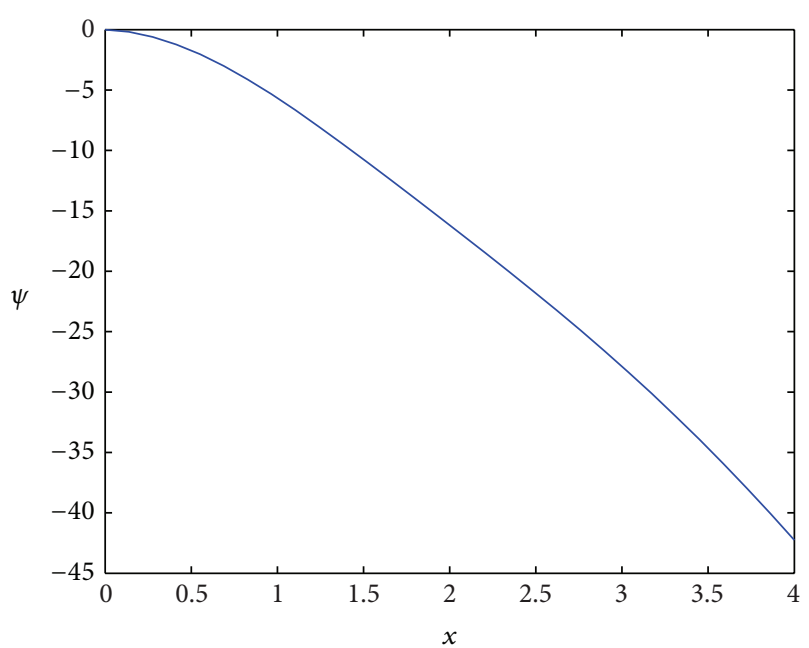

FIGURE 9: $\alpha=0.3$ and $\lambda=2$.

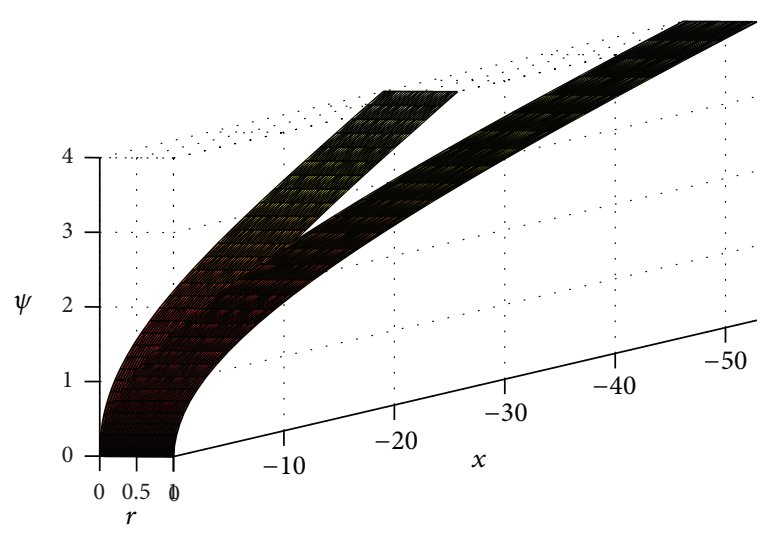

Figure 10: $\alpha=0.8$ and $\lambda=2$.

Therefore, the general solution will be as follows:

$$
\psi=(\underline{Y}(x, r), \bar{Y}(x, r)) .
$$

Figures $9,10,11$, and 12 show the graphs of the solutions for the cases of fractional, Fuzzy fractional, and fuzzy, respectively.

Example 6. Suppose the following fuzzy fractional differential equation with fuzzy initial Riemann-Liouville condition:

$$
\begin{gathered}
D_{0^{+}}^{\alpha} y(x)+\lambda y(x)=A \quad x \in R^{+} \cup 0, \lambda, A \in R^{+} \\
I_{0^{+}}^{1-\alpha} y\left(0^{+}\right)=(1+r, 3-r) .
\end{gathered}
$$

So its lower and upper functions equations are

$$
\begin{array}{r}
D_{0^{+}}^{\alpha} \underline{y}(x, r)+\lambda \underline{y}(x, r)=A \\
I_{0^{+}}^{1-\alpha} \underline{y}\left(0^{+}, r\right)=1+r, \\
D_{0^{+}}^{\alpha} \bar{y}(x, r)+\lambda \bar{y}(x, r)=A \\
I_{0^{+}}^{1-\alpha} \bar{y}\left(0^{+}, r\right)=3-r .
\end{array}
$$




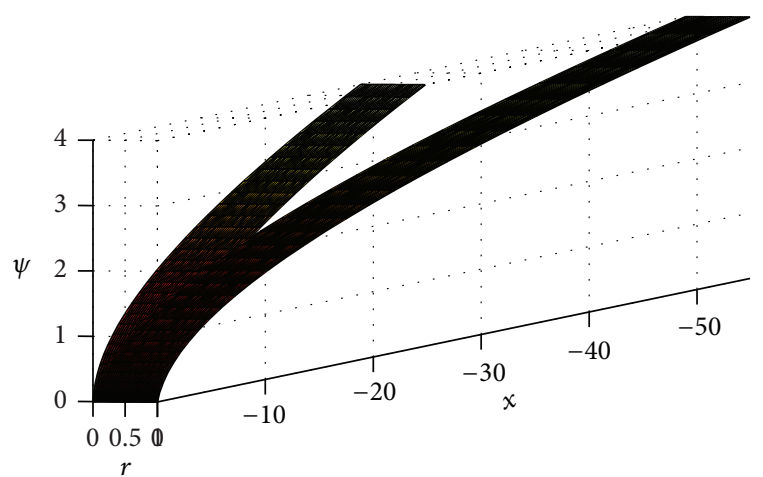

FIGURE 11: $\alpha=0.3$ and $\lambda=2$.

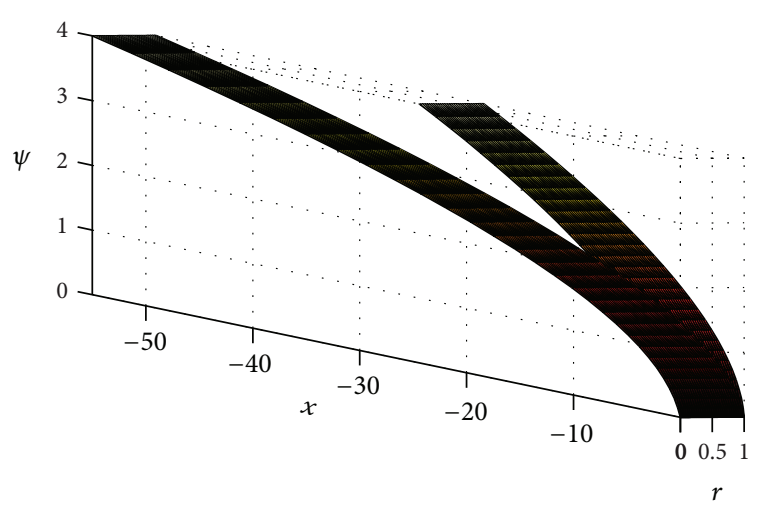

Figure 12: $\alpha=1$ and $\lambda=2$.

Using the same manner we get the solutions

$\underline{y}(x, r)=(1+r) \frac{1}{x^{1-\alpha}} E_{\alpha, \alpha}\left(-\lambda x^{\alpha}\right)+A x^{\alpha} E_{\alpha, \alpha+1}\left(-\lambda x^{\alpha}\right)$,
$\bar{y}(x, r)=(3-r) \frac{1}{x^{1-\alpha}} E_{\alpha, \alpha}\left(-\lambda x^{\alpha}\right)+A x^{\alpha} E_{\alpha, \alpha+1}\left(-\lambda x^{\alpha}\right)$.

Finally, we obtain general solution

$$
\psi=(\underline{Y}(x, r), \bar{Y}(x, r)) .
$$

Figures 13, 14, 15, and 16 indicate the graphs of the solutions for the cases of fractional, fuzzy fractional, and fuzzy, respectively.

Example 7. Let us consider the fuzzy fractional differential equation involving fuzzy Riemann-Liouville initial condition:

$$
\begin{gathered}
D_{0^{+}}^{\alpha} y(x)+\lambda y(x)=(4+r, 6-r) \\
\alpha \in(0,1), x \in R^{+} \cup 0, \lambda \in R^{+} \\
I_{0^{+}}^{1-\alpha} y\left(0^{+}\right)=(1+r, 3-r) .
\end{gathered}
$$

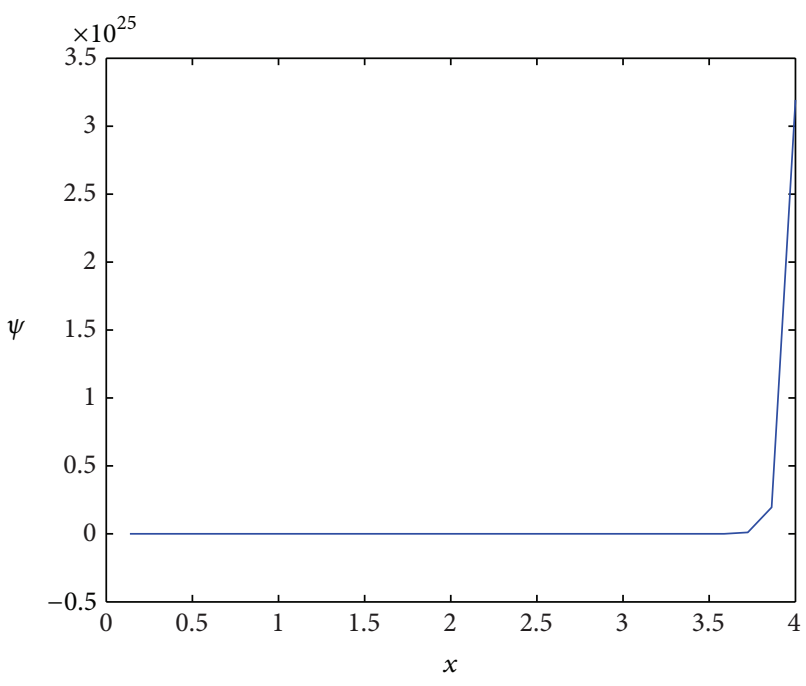

Figure 13: $\alpha=0.3$ and $\lambda=2$.

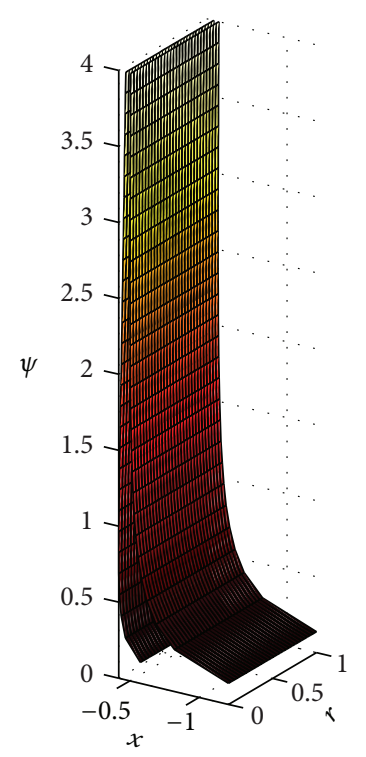

FIGURE 14: $\alpha=0.8$ and $\lambda=2$.

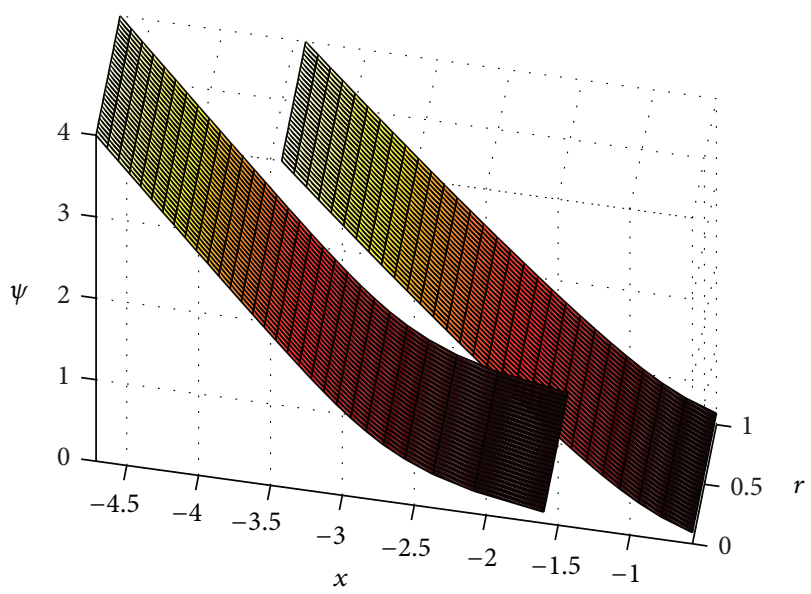

Figure 15: $\alpha=0.3$ and $\lambda=2$. 

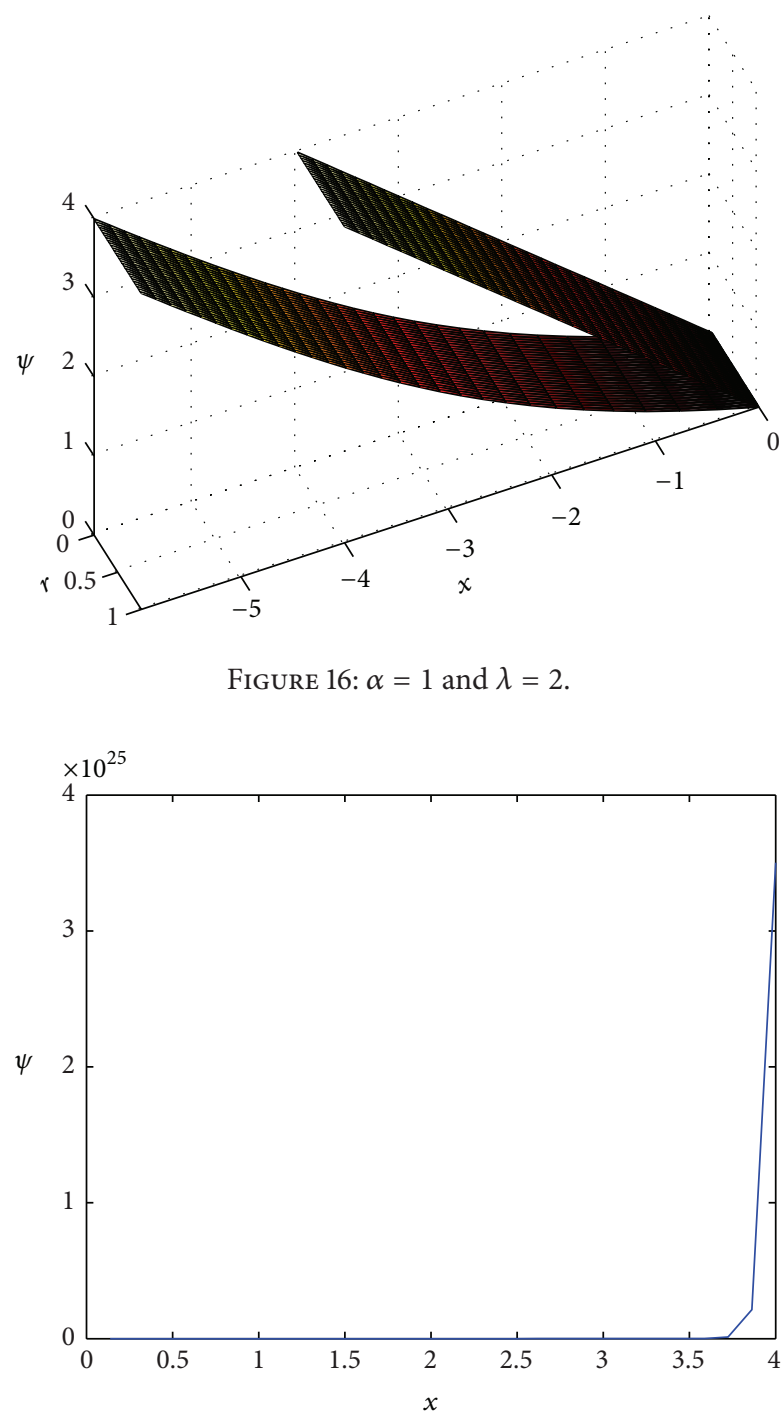

Figure 17: $\alpha=0.3$ and $\lambda=2$.

Equation (34) will be system of two equations such as

$$
\begin{gathered}
D_{0^{+}}^{\alpha} \underline{y}(x, r)+\lambda \underline{y}(x, r)=(4+r) \\
I_{0^{+}}^{1-\alpha} \underline{y}\left(0^{+}, r\right)=1+r, \\
D_{0^{+}}^{\alpha} \bar{y}(x, r)+\lambda \bar{y}(x, r)=6-r \\
I_{0^{+}}^{1-\alpha} \bar{y}\left(0^{+}, r\right)=3-r .
\end{gathered}
$$

The solutions for (34) are

$$
\begin{aligned}
\underline{y}(x, r)= & (1+r) \frac{1}{x^{1-\alpha}} E_{\alpha, \alpha}\left(-\lambda x^{\alpha}\right) \\
& +(4+r) x^{\alpha} E_{\alpha, \alpha+1}\left(-\lambda x^{\alpha}\right), \\
\bar{y}(x, r)= & (3-r) \frac{1}{x^{1-\alpha}} E_{\alpha, \alpha}\left(-\lambda x^{\alpha}\right) \\
& +(6-r) x^{\alpha} E_{\alpha, \alpha+1}\left(-\lambda x^{\alpha}\right) .
\end{aligned}
$$

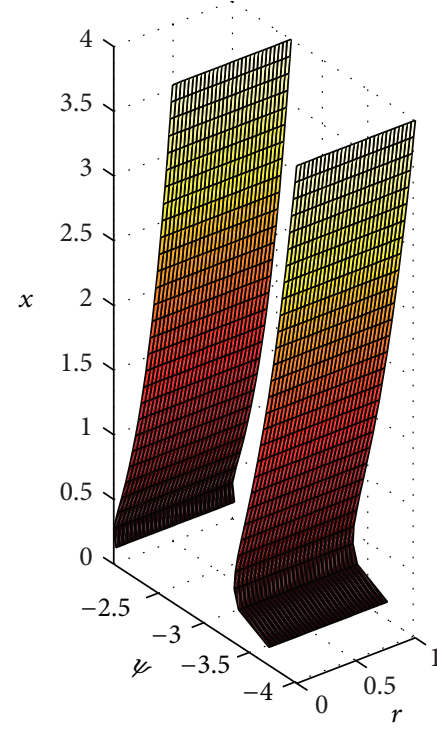

FIGURE 18: $\alpha=0.8$ and $\lambda=2$.

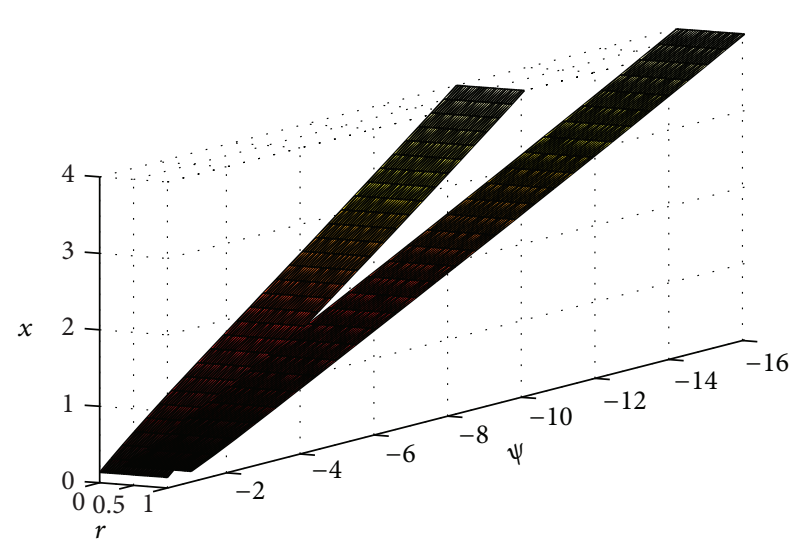

FIGURE 19: $\alpha=0.3$ and $\lambda=2$.

And the general solution will be as

$$
\psi=(\underline{Y}(x, r), \bar{Y}(x, r)) .
$$

Figures 17, 18, 19, and 20 present the graphs of the solutions for the cases of fractional, fuzzy fractional, and fuzzy, respectively.

\section{Conclusion}

In this work, we have generalized the fractional Laplace transformation to the fuzzy fractional Laplace transformation. Then, we have solved the fractional fuzzy differential equation using suggested fuzzy fractional Laplace transformation. Riemann-Liouville and Caputo fractional derivatives were used in the fractional fuzzy differential equations. Moreover, Liouville and Caputo fractional initial condition is chosen in the example to show the difference. The illustrated graphs present the difference between fuzzy, fractional, and ordinary differential equations. 


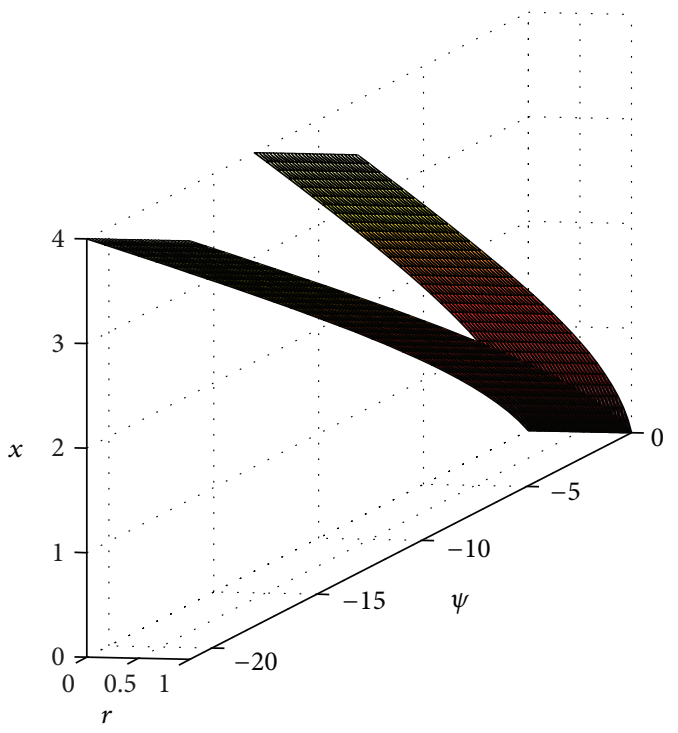

Figure 20: $\alpha=1$ and $\lambda=2$.

\section{Conflict of Interests}

The authors declare that there is no conflict of interests regarding the publication of this paper.

\section{References}

[1] K. B. Oldham and J. Spanier, The Fractional Calculus, Academic Press, New York, NY, USA, 1974.

[2] K. S. Miller and B. Ross, An Introduction to the Fractional Calculus and Fractional Differential Equations, John Wiley \& Sons, New York, NY, USA, 1993.

[3] S. G. Samko, A. A. Kilbas, and O. I. Marichev, Fractional Integrals and Derivatives, Gordon and Breach Science Publishers, New York, NY, USA, 1993.

[4] R. Gorenflo and F. Mainardi, "Fractional calculus: integral and differential equations of fractional order," in Fractals and Fractional Calculus in Continuum Mechanics, pp. 223-276, Springer, New York, NY, USA, 1997.

[5] I. Podlubny, Fractional Differential Equations, vol. 198 of Mathematics in Science and Engineering, Academic Press, New York, NY, USA, 1999.

[6] R. Hilfer, Applications of Fractional Calculus in Physics, World Scientific, 2000.

[7] B. J. West, M. Bologna, and P. Grigolini, Physics of Fractal Operators, Springer, New York, NY, USA, 2003.

[8] G. M. Zaslavsky, Hamiltonian Chaos and Fractional Dynamics, Oxford University Press, 2008.

[9] A. A. Kilbas, H. M. Srivastava, and J. J. Trujillo, Theory and Applications of Fractional Differential Equations, vol. 204 of North-Holland Mathematics Studies, Elsevier, Amsterdam, The Netherlands, 2006.

[10] A. K. Golmankhaneh, Investigations in Dynamics: with Focus on Fractional Dynamics, Lap Lambert Academic Publishing, 2012.

[11] R. Herrmann, Fractional Calculus an Introduction for Physicists, World Scientific Publishing, 2011.
[12] D. Baleanu, K. Diethelm, E. Scalas, and J. J. Trujillo, Fractional Calculus Models and Numerical Methods, World Scientific Publishing, 2012.

[13] D. Baleanu, J. A. T. Machado, and A. C. Luo, Fractional Dynamics and Control, Springer, 2012.

[14] A. K. Golmankhaneh, N. A. Porghoveh, and D. Baleanu, "Mean square solution of second-order random differential equations by using Homotopy analysis method," Romanian Reports in Physics, vol. 65, no. 2, pp. 350-361, 2013.

[15] H. Jafari, H. Tajadodi, D. Baleanu, A. A. AL-Zahrani, Y. A. AL Hamed, and A. H. Zahid, "Exact solution of boussinesq and Kdv-mKdv equations by fractional sub-equation method," Romanian Reports in Physics, vol. 65, no. 4, pp. 1119-1124, 2013.

[16] A. Parvate and A. D. Gangal, "Calculus on fractal subsets of real line-II: conjugacy with ordinary calculus," Fractals, vol. 19, no. 3, pp. 271-290, 2011.

[17] X.-J. Yang, Advanced Local Fractional Calculus and Its Applications, World Science, New York, NY, USA, 2012.

[18] X.-J. Yang, Local Fractional Functional Analysis and Its Applications, Asian Academic Publisher Limited, Hong Kong, 2011.

[19] R. Goetschel Jr. and W. Voxman, "Elementary fuzzy calculus," Fuzzy Sets and Systems, vol. 18, no. 1, pp. 31-43, 1986.

[20] V. Lakshmikantham and R. N. Mohapatra, Theory of Fuzzy Differential Equations and Inclusions, vol. 6 of Series in Mathematical Analysis and Applications, CRC Press, 2003.

[21] P. Diamond and P. Kloeden, Metric Spaces of Fuzzy Sets: Theory and Applications, World Scientific, Singapore, 1994.

[22] J. J. Buckley, E. Eslami, and T. Feuring, Fuzzy Mathematics in Economics and Engineering, vol. 91 of Studies in Fuzziness and Soft Computing, Physica, Heidelberg, Germany, 2002.

[23] B. Bede, I. J. Rudas, and A. L. Bencsik, "First order linear fuzzy differential equations under generalized differentiability," Information Sciences, vol. 177, no. 7, pp. 1648-1662, 2007.

[24] R. P. Agarwal, V. Lakshmikantham, and J. J. Nieto, "On the concept of solution for fractional differential equations with uncertainty," Nonlinear Analysis: Theory, Methods \& Applications, vol. 72, no. 6, pp. 2859-2862, 2010.

[25] O. Kaleva, "Fuzzy differential equations," Fuzzy Sets and Systems, vol. 24, no. 3, pp. 301-317, 1987.

[26] A. Khastan and J. J. Nieto, "A boundary value problem for second order fuzzy differential equations," Nonlinear Analysis: Theory, Methods \& Applications, vol. 72, no. 9-10, pp. 3583-3593, 2010.

[27] A. Salah, M. Khan, and M. A. Gondal, "A novel solution procedure for fuzzy fractional heat equations by homotopy analysis transform method," Neural Computing and Applications, vol. 23, no. 2, pp. 269-271, 2013.

[28] S. Arshad and V. Lupulescu, "Fractional differential equation with the fuzzy initial condition," Electronic Journal of Differential Equations, vol. 34, pp. 1-8, 2011.

[29] S. Salahshour, T. Allahviranloo, and S. Abbasbandy, "Solving fuzzy fractional differential equations by fuzzy Laplace transforms," Communications in Nonlinear Science and Numerical Simulation, vol. 17, no. 3, pp. 1372-1381, 2012.

[30] G. A. Anastassiou, Fuzzy Mathematics: Approximation Theory, vol. 251 of Studies in Fuzziness and Soft Computing, Springer, 2010.

[31] G. A. Anastassiou, "Fuzzy fractional calculus and the Ostrowski integral inequality," in Intelligent Mathematics: Computational Analysis, vol. 5 of Intelligent Systems Reference Library, pp. 553574, Springer, Berlin, Germany, 2011. 
[32] G. A. Anastassiou, "Fuzzy Ostrowski inequalities," in Fuzzy Mathematics: Approximation Theory, vol. 251 of Studies in Fuzziness and Soft Computing, pp. 65-73, Springer, Berlin, Germany, 2010.

[33] L. Kexue and P. Jigen, "Laplace transform and fractional differential equations," Applied Mathematics Letters, vol. 24, no. 12, pp. 2019-2023, 2011.

[34] S. Arshad and V. Lupulescu, "On the fractional differential equations with uncertainty," Nonlinear Analysis: Theory, Methods \& Applications, vol. 74, no. 11, pp. 3685-3693, 2011.

[35] M. Mazandarani and A. V. Kamyad, "Modified fractional Euler method for solving fuzzy fractional initial value problem," Communications in Nonlinear Science and Numerical Simulation, vol. 18, no. 1, pp. 12-21, 2013. 


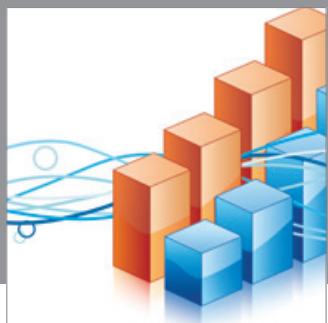

Advances in

Operations Research

mansans

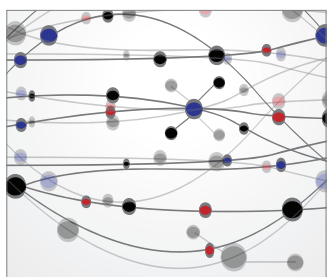

The Scientific World Journal
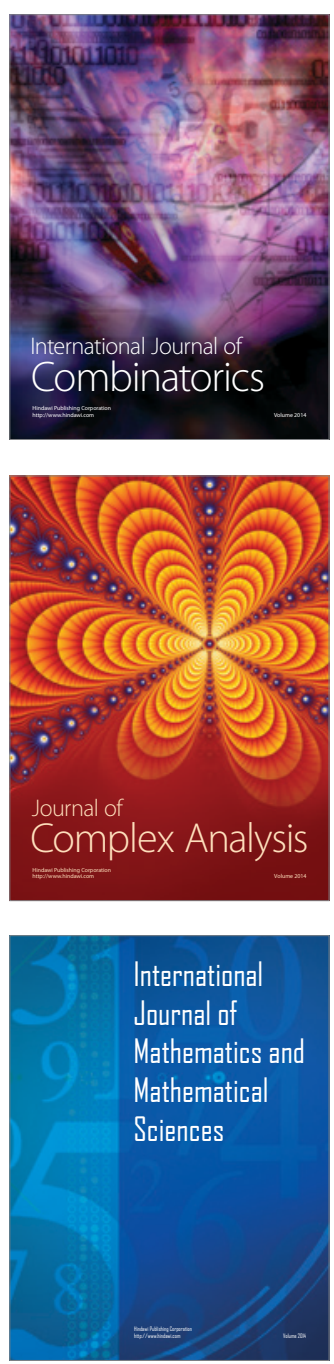
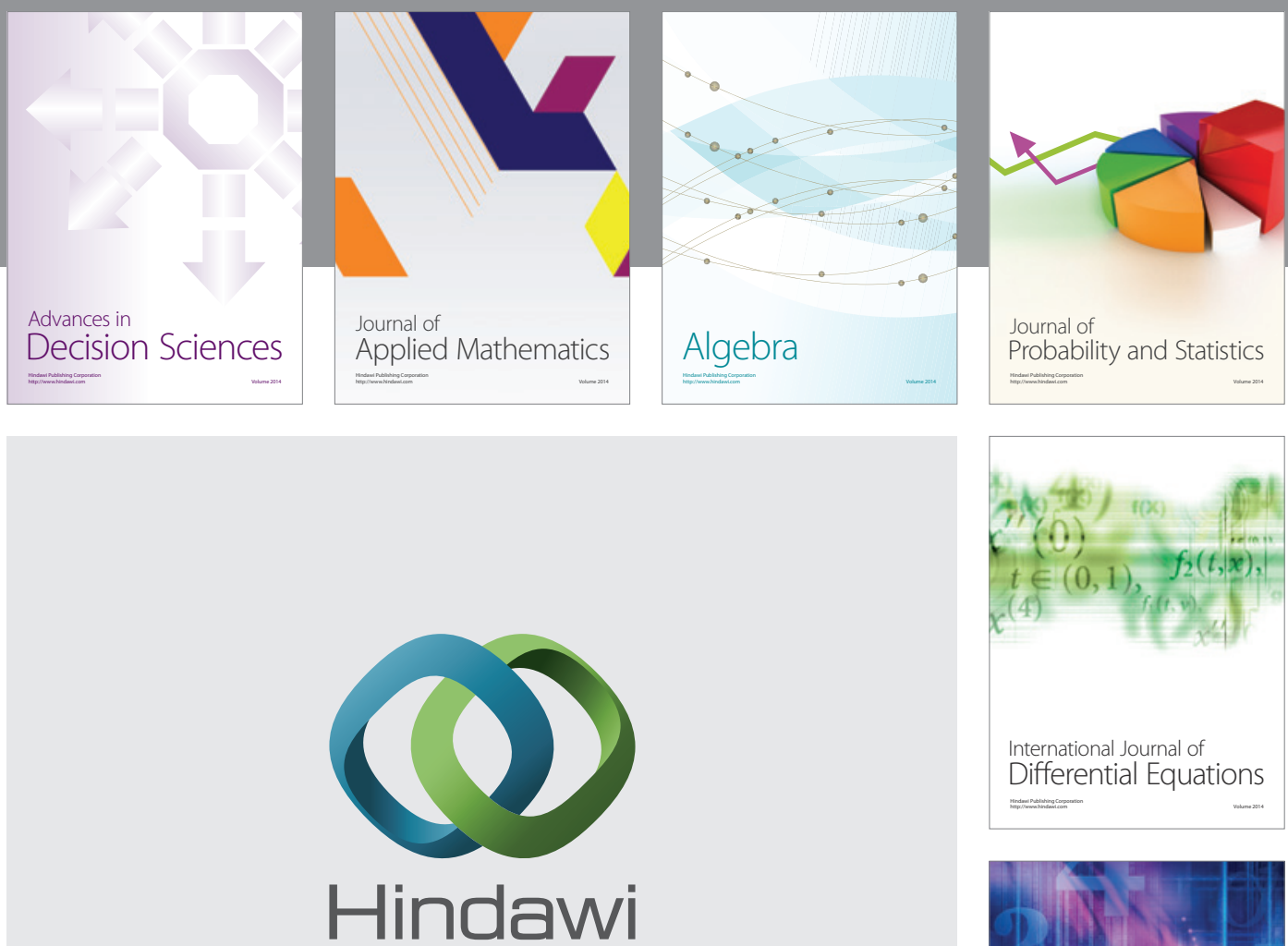

Submit your manuscripts at http://www.hindawi.com
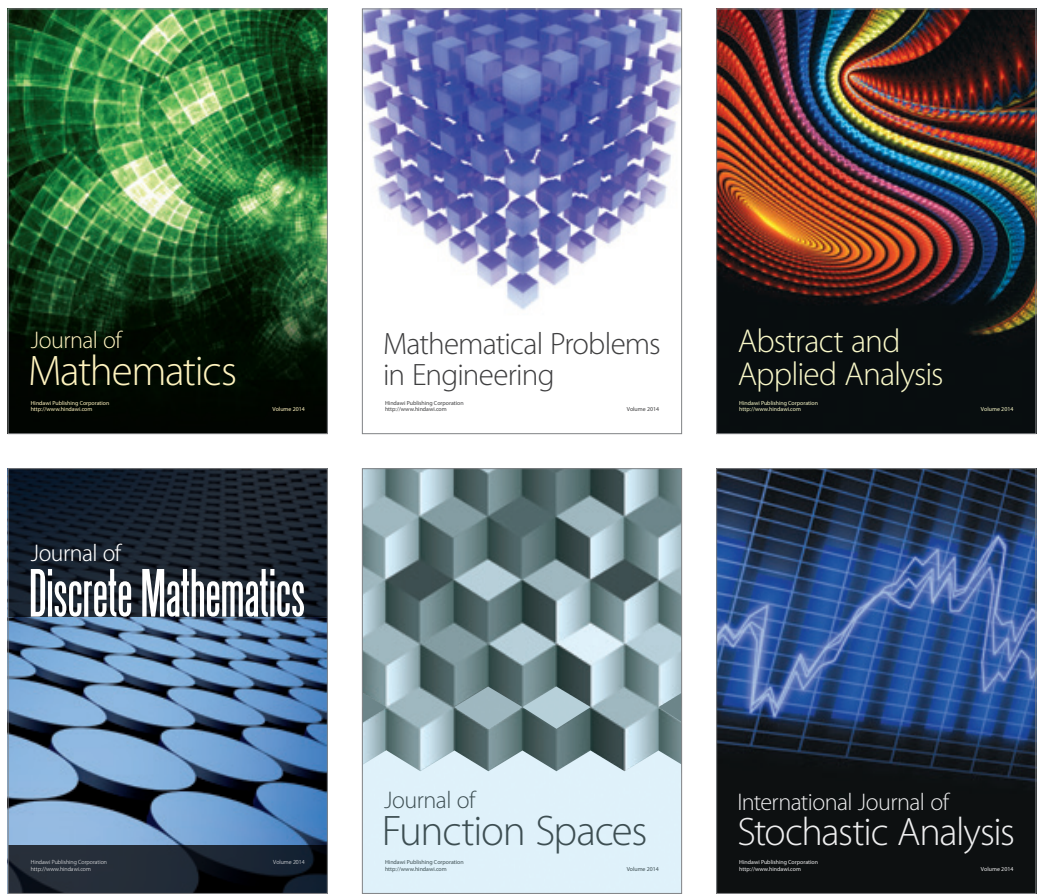

Journal of

Function Spaces

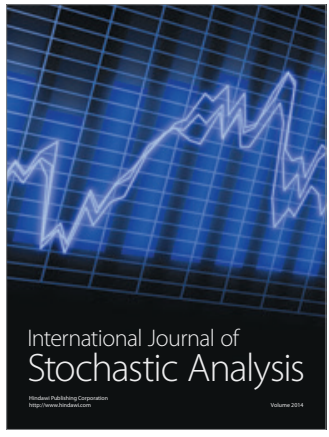

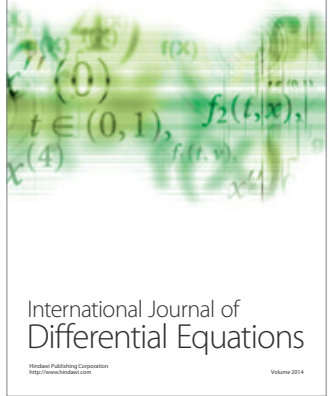
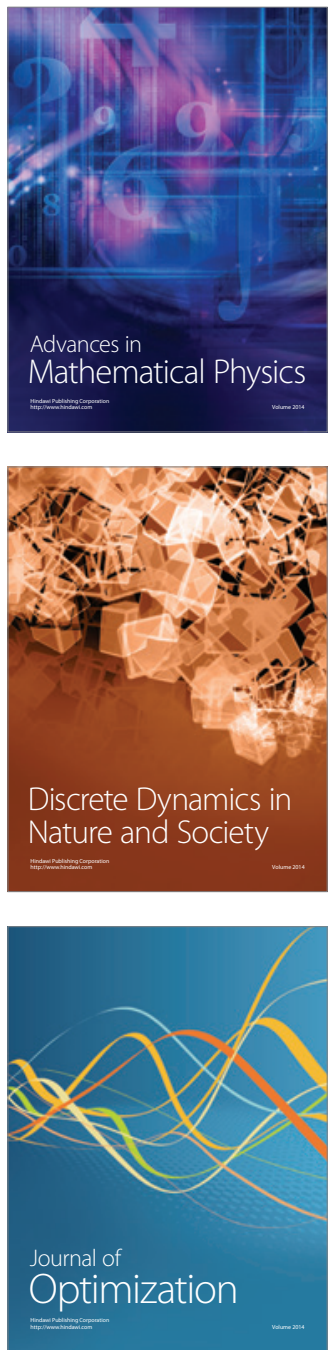